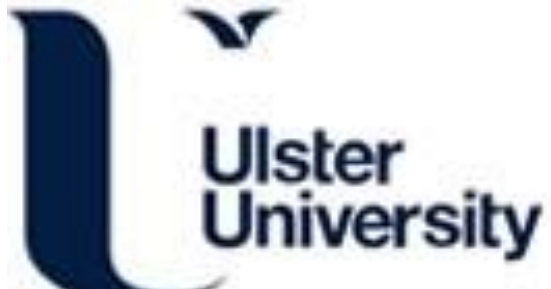

Code-to-code verification of an axisymmetric model of the Bridgman solidification process for alloys.

Seredyński, M., Battaglioli, S., Mooney, R. P., Robinson, A. J., Banaszek, J., \& McFadden, S. (2017). Code-tocode verification of an axisymmetric model of the Bridgman solidification process for alloys. International Journal of Numerical Methods for Heat and Fluid Flow, 27(5). https://doi.org/10.1108/HFF-03-2016-0123

Link to publication record in Ulster University Research Portal

\section{Published in:}

International Journal of Numerical Methods for Heat and Fluid Flow

Publication Status:

Published (in print/issue): 02/05/2017

DOI:

10.1108/HFF-03-2016-0123

\section{Document Version}

Author Accepted version

\section{General rights}

Copyright for the publications made accessible via Ulster University's Research Portal is retained by the author(s) and / or other copyright owners and it is a condition of accessing these publications that users recognise and abide by the legal requirements associated with these rights.

\section{Take down policy}

The Research Portal is Ulster University's institutional repository that provides access to Ulster's research outputs. Every effort has been made to ensure that content in the Research Portal does not infringe any person's rights, or applicable UK laws. If you discover content in the Research Portal that you believe breaches copyright or violates any law, please contact pure-support@ulster.ac.uk. 


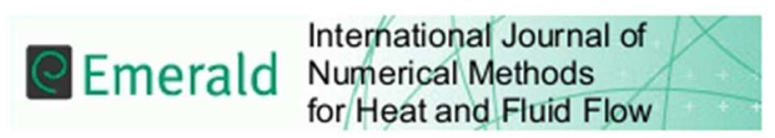

\section{Code-to-code verification of an axisymmetric model of the Bridgman solidification process for alloys.}

\begin{tabular}{|r|l|}
\hline Journal: & International Journal of Numerical Methods for Heat and Fluid Flow \\
\hline Manuscript ID & HFF-03-2016-0123.R2 \\
\hline Manuscript Type: & Research Article \\
\hline Keywords: & Bridgman furnace, solidification, code verification \\
\hline \multicolumn{2}{|l}{} \\
\hline
\end{tabular}

SCHOLARONE ${ }^{\text {m }}$

Manuscripts 


\title{
Code-to-code verification of an axisymmetric model of the Bridgman solidification process for alloys
}

Keywords: Bridgman furnace; solidification; code verification

\begin{abstract}
Numerical models of manufacturing processes are useful and provide insight for the practitioner, however, model verification and validation are a prerequisite for expedient application. This paper details the code-to-code verification of a thermal numerical model for the Bridgman solidification process of alloys in a 2-dimensional axisymmetric domain, against an established commercial code (ANSYS Fluent); the work is considered a confidence building step in model development. A grid sensitivity analysis is carried out to establish grid independence, this is followed by simulations of two transient solidification scenarios: pulling rate step change and ramp input; the results of which are compared and discussed. Good conformity of results is achieved, hence the non-commercial model is codeto-code verified; in addition, the ability of the non-commercial model to deal with radial heat flow is demonstrated. The introduction of front tracking to model the macroscopic growth of dendritic mush and the region of undercooled liquid is identified as the next step in model development.
\end{abstract}

\section{Introduction}

A validated numerical model of a manufacturing process is a useful tool since it gives the practitioner deeper insight into the workings of the process. Solidification processing of metallic alloys has benefited from the application of numerical models in two ways. Firstly, numerical models have been used to simulate a process before the real application of the process; hence, assisting with the planning stage. Secondly, since most microstructural characterisation techniques are performed post-mortem, modelling has often been used in conjunction with measured transient data from an experiment to simulate the actual transient conditions that occurred during the process. 
As part of model development, a numerical model should be verified before it is validated. Validation is achieved by confirming agreement between numerical model simulations and well-understood benchmark experiments. Verification (a prerequisite to validation) is preferably achieved by comparing numerical model simulation results against a closed-form analytical (exact) solution. However, such closed-form solutions are often difficult or impossible to obtain. In the case where an analytical solution is unavailable, numerical modelling simulation results can be compared to alternative or established numerical models of the process; this approach is known as 'code-to-code' verification and is the subject of this manuscript. It is important to note that while this approach is useful, complete Verification of Code can only be achieved using an exact analytical model (Pelletier and Roache, 2000). Verification of Calculations can be completed using formal order of accuracy methods, where the difference between numerical solutions at different grid resolutions is used to confirm that the designed order of accuracy of the discretisation scheme is achieved. The code-to-code approach is essentially a confidence building exercise in the overall model development process, as part of the verification step.

This manuscript focuses on the numerical modelling of the Bridgman solidification process, a process used widely in industry and research. Many authors, on account of its attractive method where temperature gradient and growth rate can be independently controlled, have applied Bridgman solidification experimentally. For example, the Bridgman solidification technique has recently been applied in experiments carried out in the Materials Science Laboratory on-board the International Space Station (Liu et al., 2014). At a more practical level, Bridgman furnace based experiments are commonplace in literature, for example, the studies of Mooney et al. (2014) and Rosch et al. (1993) endeavour to accurately determine the furnace heat transfer coefficients (an important parameter for modelling). 


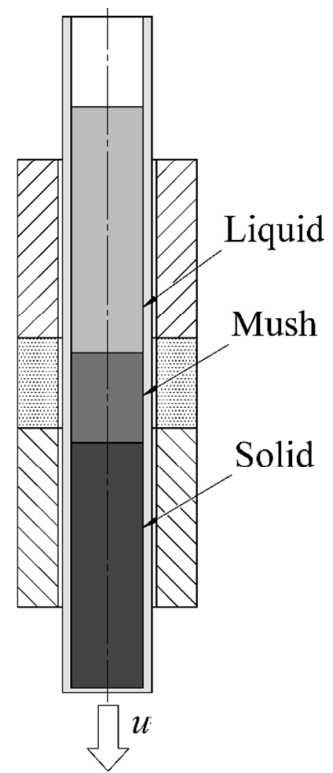

Figure 1: Schematic diagram and cross-section of a Bridgman furnace, crucible, and sample.

The Bridgman furnace, figure 1, is used to control the directional solidification of a sample material inside a moving crucible. Two temperature controlled elements, a heater and a crystalliser, are separated by an adiabatic baffle zone. The heater is held at a temperature above the liquidus temperature, thus creating a hot zone; the crystalliser is held at a temperature below the solidus (or eutectic) temperature, thus creating a cold zone; and solidification normally occurs within these zones. This furnace setup enables the user to establish a thermal gradient, $G$, in the axial direction of the sample. Concurrently, the crucible and sample assembly are translated towards the cold zone with a pulling speed, $u$, thus ensuring that solidification occurs at a controlled cooling rate, since the cooling rate of the solidification process can be estimated as the product of gradient $G$ and speed $u$. In alloy materials (that exhibit a freezing range) solidification proceeds with the development of a semi-solid region between the liquid and solid phases, known as the mushy zone. Latent heat is released from within the mushy zone as the portion of solid within the mush ('solid fraction') increases.

Typically, a Bridgman furnace is operated in steady-state mode with the temperature gradient and pulling speed held constant. Steady-state solidification is the simplest mode of operation for the furnace. A steady-state model of the furnace may be used to infer difficult-to-measure parameters, for example, thermal gradients at the solid-liquid interfaces, axial temperature profile, and the position of the mushy zone. However, a Bridgman furnace may also be operated in transient modes. The transient modes of operation can be realised by changing, in 
a controlled manner over time, the temperature of the heaters, the pulling speed, or both heater temperature and pulling speed simultaneously. In such operating modes the thermal conditions within the sample are transient. These conditions are required, for instance, when phenomena like the columnar to equiaxed transition are investigated. Several experimental studies may be found in literature on the matter e.g. Jung et al. (2009) and Zhong et al. (2015). However, the opaque nature of metallic alloys does not allow for direct observation of transient solidification, and although some advanced techniques like X-ray radiographic imaging can sometimes be used to monitor the real-time evolution of the microstructure (Reinhart et al., 2005), post mortem analysis is often employed. Therefore, a transient numerical model of the furnace is required to estimate the actual thermal conditions in the solidifying sample as a function of time.

Several models have been developed for this purpose. Timchenko et al. (2002, 2000) employed a single domain enthalpy method for Bridgman solidification, valid when isothermal phase change could be assumed, i.e. for conditions when no mushy zone develops between solid and liquid phases. Kartavykh et al. (2014) implemented a numerical model that simulated the mushy zone evolution in TiAl-based alloys during power-down solidification (i.e. varying heaters temperatures), but without the sample translation.

The Bridgman Furnace Front Tracking Model (BFFTM) is a thermal numerical model developed by Mooney et al. (2012) that uses a 1-dimensional (1D) finite-difference control volume approach. The model allows simulating the development of the columnar grain region in the sample for operating modes with both varying pulling speed and varying heaters temperature. The 'front tracking' method used is based on that developed by Browne and Hunt (2004) and later extended by McFadden and Browne (2009) for fixed grid, fixed sample problems. The original model of Browne and Hunt was later generalized to cases involving thermal convection (Banaszek et al., 2007); experimental data was obtained for a Bridgman solidification process in power-down mode for the purposes of model validation. In this case, the heat transfer coefficient was unknown; instead a single cooling curve was used to calibrate the model. A similar concept, utilizing the tracking a moving envelope of columnar dendrite tips on a fixed control volume grid was developed by Seredyński and Banaszek $(2010,2012)$ to identify the regions in the slurry zone during metal alloy solidification. A similar front tracking approach to modelling the solidification of semi-transparent materials in Bridgman furnaces was also developed by Łapka and Furmański (2012). 
The BFFTM has been subjected to a formal 'verification of code' analysis where the solidification of a pure material in a Bridgman furnace was simulated (Mooney and McFadden, 2014). Furthermore, the BFFTM has been applied in a steady-state scenario (Mooney et al., 2014) to determine Bridgman furnace heat transfer coefficients; in transient scenarios to analyse axial and radial heat transfer (Mooney, Hecht, et al., 2015); and in a study focused on the columnar to equiaxed transition in gamma-TiAl alloys (Mooney et al., 2015).

Due to the 1D model construction, the BFFTM of Mooney et al. is restricted to cases where the Biot number is low $(\mathrm{Bi}<0.1)$. In the cases where the Biot number is higher than 0.1 , the radial temperature gradients may become significant and non-planar isotherms will be present within the sample. A new Bridgman model is under development that deals with larger Biot numbers (Battaglioli et al., 2015). This new model of Bridgman solidification uses a twodimensional (2D) axisymmetric geometry and a finite-volume approach, and like the BFFTM allows to simulate directly the translation of the sample. Because the new model is under development, it is an imperative to build confidence in the code; hence, in this manuscript the 2D axisymmetric model is verified against a commercial code to obtain a code-to-code verification.

\subsection{Aims and objectives}

To develop confidence in the development of a 2D axisymmetric model of the Bridgman process, the following aims and objectives are stated:

1. Provide an overview of the construction of the new Bridgman furnace model.

2. Develop an alternative model of benchmark simulations using a commercial software package.

3. Perform a grid sensitivity analysis to demonstrate mesh independence.

4. Compare results from each model over a range of suitable simulation scenarios.

5. Demonstrate the efficacy of the new Bridgman furnace model for cases with significant radial heat transfer.

Given this introduction section, this manuscript is divided into the following sections: Methodology, Modelling and Material Data, Results, Discussion, Conclusion, and Acknowledgements.

The 'Methodology' section describes the new Bridgman model ('non-commercial model') that uses the finite-volume approach and the model developed within a commercial package 
('commercial model'). The commercial package used is ANSYS Fluent 15 with specific, non-proprietary, details applied via a User Defined Function (UDF).

The 'Modelling and Material Data' section includes details of the alloy materials used in this analysis and the thermophysical properties required to run the models. Two alloy materials have been selected for this study, namely, Al-11wt.\%Si and Al-7wt.\%Si. These alloys were selected because they are hypo-eutectic alloys, data for which is readily available from literature. Initial and boundary conditions are described in this section along with key simulation input data.

The 'Results' section shows the thermal data from each model so that direct comparisons can be made between the simulation results for each model. A 'Discussion' section follows the results section; and finally, a 'Conclusion' section reviews the outcomes of the study and summarises the main findings. Future directions in the development of the non-commercial model are also outlined in the conclusion section.

\section{Methodology}

\subsection{General description}

The geometry of the Bridgman furnace is comparable with that considered by Mooney et al. (Mooney et al., 2014, 2012) and Battaglioli et al. (Battaglioli et al., 2015). Figure 2 shows the model geometry; the high-temperature and low-temperature zones are separated by an adiabatic baffle zone that is set in the central part of the furnace. The length of the adiabatic zone is given by the distance $s$. The length of the high temperature heater is $x_{H}$ and the total length of the computational domain is $l$. The overall radius of the sample is given as $r_{s}$.

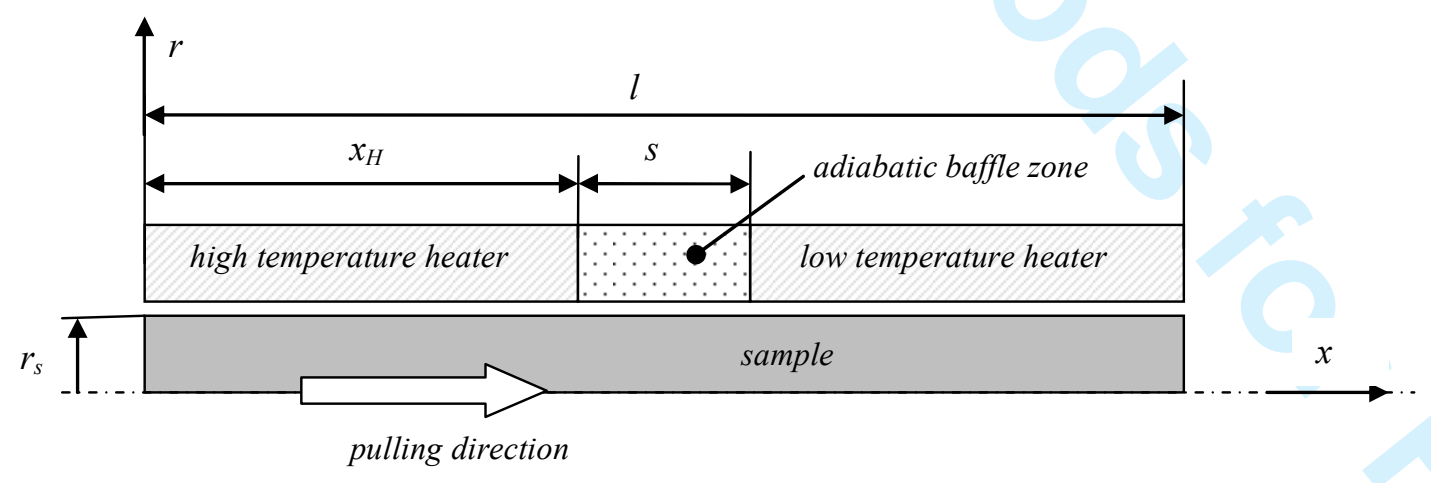

Figure 2: Bridgman furnace model geometry.

The cylindrical co-ordinates $r$ and $x$ are used for the radial position and axial position, respectively. Because the computational domain is symmetric about the $x$-axis, only half of 


$$
\frac{\partial\left(\rho C_{p} T\right)}{\partial t}=\frac{1}{r} \frac{\partial}{\partial r}\left(r k \frac{\partial T}{\partial r}\right)+\frac{\partial}{\partial x}\left(k \frac{\partial T}{\partial x}\right)-u \frac{\partial\left(\rho C_{p} T\right)}{\partial x}+\rho u L \frac{\partial g_{s}}{\partial x}+\rho L \frac{\partial g_{s}}{\partial t}
$$

where $\rho$ is density; $c_{p}$, specific heat; $T$, temperature; $t$, time; $k$, thermal conductivity; $u$, pulling velocity; $L$, latent heat per unit mass; and $g_{S}$, volumetric solid fraction. The term on the left hand side of equation (1) is the sensible heat term. The first two terms on the right hand side (RHS) of the equation deal with heat due to conduction in the radial and axial directions, respectively. The third term on the RHS is due to advection of sensible heat in the axial direction. The fourth term on the RHS is due to advection of latent heat in the mushy zone and, finally, the fifth term on the RHS is due the release of latent heat as the solid fraction increases over time. Convective transport is driven only by the bulk movement of the sample through the fixed domain due to pulling; thermo-solutal convection is neglected in this model. (The solid and liquid phases are assumed to move at the same rate.)

Solidification is assumed to follow the Scheil (1942) microsegregation rule; hence, for a hypoeutectic binary alloy, solid fraction may be given as a function of temperature as follows:

$$
g_{s}(T)=\left\{\begin{array}{cc}
0 & T \geq T_{L} \\
1-\left(\frac{T_{M}-T}{T_{M}-T_{L}}\right)^{1 /\left(k_{p}-1\right)} & T_{E}<T<T_{L} \\
1 & T \leq T_{E}
\end{array}\right.
$$

where $k_{p}$ is the partition coefficient; $T_{M}$, the melting temperature of pure alloy; $T_{L}$, the equilibrium liquidus temperature of the alloy; and $T_{E}$, the eutectic temperature. Since equation (2) is non-linear, a Newton-Raphson iterative scheme is used to calculate the solid fraction at each time step of the numerical scheme. Note that isothermal solidification is assumed at the eutectic temperature, i.e., when $T=T_{E}$. The numerical treatment of both Scheil and isothermal eutectic solidification is provided in detail elsewhere (McFadden and Browne, 2009). 
The computational domain (which covers half of the axial cross section of the sample) is discretized with a uniform and orthogonal control volume mesh. The $2 \mathrm{D}$ mesh has grid spacings of $\Delta r$ and $\Delta x$ in the radial and axial directions, respectively. The control volumes in the $2 \mathrm{D}$ axisymmetric scheme are rectangular cross-section toroids. An explicit finitedifference scheme is used. Hence, an algebraic equation is developed which is used to determine the temperatures of each control volume at time intervals separated by $\Delta t$ seconds. As is typical with an explicit numerical scheme, the selected time step must provide a stable and converged solution. The Péclet numbers for the simulations presented in this manuscript are less than unity. Hence, there is no requirement for an upwind scheme. Greater detail on the derivation of the numerical scheme is provided elsewhere (Battaglioli et al., 2015).

\subsection{Adapted commercial model (commercial code)}

For code-to-code verification purposes, ANSYS Fluent 15 (a commercially established software) was used to model the Bridgman process. However, the model had to be adapted with User Defined File (UDF) macros to reflect the general problem as described in Section 2.1. Specifically, the Scheil rule for alloy solidification had to be implemented into the commercial code. The specific heat capacity and enthalpy were defined using the DEFINE_SPECIFIC_HEAT type macro, and the non-linear solid fraction to temperature relation was implemented within it. In order to prevent oscillatory solutions during the eutectic transformation, an artificially high but finite slope was assumed for the solid fraction versus temperature relationship when close to the eutectic temperature, $T_{E}$. The non-iterative PISO scheme was applied to solve the equation with a time step $\Delta t$. To simulate the pulling velocity in the whole domain, the shear stress equal to $0 \mathrm{~Pa}$ at circumferential walls was imposed.

\section{Modelling and Material Data}

This section contains information regarding all of the simulation inputs.

\subsection{Material properties}

As previously mentioned, the alloy systems selected for modelling are Al-11wt.\%Si and Al$7 \mathrm{wt} . \% \mathrm{Si}$. The thermophysical properties of these alloys vary with temperature; the data for which are found elsewhere (McFadden et al., 2009). In the mushy zone, a rule of mixing was applied that calculates the thermophysical properties as a function of solid fraction (McFadden and Browne, 2009). 


\subsection{Initial and boundary conditions}

The boundaries to the system are identifiable from figure 2. The following Dirichlet boundary conditions are applied at the two ends of the sample domain:

$$
\begin{aligned}
& T(x, r, t)=T_{\text {Left }}, \quad x=0,0<r<r_{s}, t \geq 0 \\
& T(x, r, t)=T_{\text {Right }}, \quad x=l, 0<r<r_{s}, \quad t \geq 0 .
\end{aligned}
$$

The temperature $T_{\text {Left }}$ is the temperature setting at the left boundary where $x=0$; whereas, $T_{\text {Right }}$ is the temperature setting at the right boundary, $x=L$. Furthermore, throughout this manuscript the following settings are applied $T_{\text {Left }}=T_{H}$ and $T_{\text {Right }}=T_{C}$. The boundary condition along the circumferential boundary changes according to three sections: the high temperature heater, the adiabatic baffle, and the low temperature heater. A third kind (Robin type) boundary condition is imposed in the high temperature section using a convection heat transfer coefficient, $h$, to give the heat flux at the boundary as follows:

$$
q(x, r, t)=-h\left(T_{w}-T_{H}\right), \quad 0<x<x_{H}, \quad r=r_{s}, \quad t \geq 0
$$

Where $q(x, r, t)$ is the heat flux at the sample wall (at the circumference of the sample), $T_{H}$ is the heater temperature, and $T_{w}$ is the temperature at the sample wall, $T_{w}=T\left(x, r_{s}\right)$. In this case the heater is usually a heat source, i.e., heat flows into the sample. Similarly for the low temperature region, a third kind (Robin type) boundary condition is imposed as follows:

$$
q(x, r, t)=-h\left(T_{w}-T_{C}\right), \quad\left(x_{H}+s\right)<x<l, \quad r=r_{s}, \quad t \geq 0 .
$$

Hence, in the low temperature section of the heater, the heat flux, $q$, depends on the heat transfer coefficient, $h$, and the low temperature heater temperature, $T_{C}$. (This region of the furnace can be a heat source or a heat sink depending on the direction of the heat flux.)

A second kind (Neumann type) boundary condition, or more specifically an adiabatic boundary condition, is applied at the baffle zone as follows:

$$
q(x, r, t)=0, \quad x_{H}<x<\left(x_{H}+s\right), r=r_{s}, t \geq 0 .
$$

Finally, due to symmetry, an adiabatic boundary condition is assumed at the axis of the sample.

$$
q(x, r, t)=0, \quad 0<x<l, r=0, t \geq 0
$$


The initial temperature profile, $T_{o}(x, r)$, is assumed to be a piecewise linear function over the three domain sections as follows:

$$
T_{o}(x, r)=\left\{\begin{array}{cc}
T_{H} & 0<x<x_{H}, 0<r<r_{s} \\
T_{H}-\left(T_{H}-T_{C}\right)\left(x-x_{H}\right) / s & x_{H}<x<\left(x_{H}+s\right), 0<r<r_{s} \\
T_{C} & \left(x_{H}+s\right)<x<l, 0<r<r_{s}
\end{array}\right.
$$

Since this initial condition is physically unrealistic (but numerically convenient), all simulations begin with an initial settling phase where the pulling speed is held at zero temporarily. After this initial settling phase, the temperature profile reaches an equilibrium profile that is equivalent to the initial temperature profile in a Bridgman furnace experiment. The temperature profile after the settling phase is considered as an improved initial condition which has reached a steady condition.

\subsection{Simulation data}

The following physical process parameters were chosen for the proceeding analysis. The process parameters relate to the geometry of the furnace, the temperatures of the heaters, and the pulling speed time profiles. Table 1 provides the details on the physical parameters that relate to the geometrical and thermal settings of the furnace. Note that the heater temperature $T_{H}$ is consistently set $50^{\circ} \mathrm{C}$ higher than the equilibrium liquidus temperature of the alloy.

Table 1: Physical process parameters inputs: geometry and temperature settings.

\begin{tabular}{lcc}
\hline Physical process parameter [units] & Symbol & Value \\
\hline Overall sample length $[\mathrm{mm}]$ & $l$ & 200 \\
High temperature heater length $[\mathrm{mm}]$ & $x_{H}$ & 60 \\
Adiabatic baffle length $[\mathrm{mm}]$ & $S$ & 30 \\
Sample radius $[\mathrm{mm}]$ & $r_{s}$ & 16 \\
High temperature heater setting $\left[{ }^{\circ} \mathrm{C}\right]$ & $T_{H}$ & $T_{L}+50$ \\
Low temperature heater setting $\left[{ }^{\circ} \mathrm{C}\right]$ & $T_{C}$ & 527 \\
Heat transfer coefficient $\left[\mathrm{W} / \mathrm{m}^{2} /{ }^{\circ} \mathrm{C}\right]$ & $h$ & 1500 \\
\hline
\end{tabular}

The analysis provided here is transient since the pulling speed varies with time. Two pulling regimes were applied to the model: velocity step input (also called a velocity jump) and a velocity ramp input. The velocity jump is the most abrupt input change possible and is often used in practice to promote Columnar to Equiaxed Transition in the alloy's grain structure (Reinhart et al., 2005). The velocity step input used in this analysis is a double step input: and is characterised as follows: 
The parameters $u_{1}$ and $u_{2}$ are the absolute pulling speed values after each step change. The time parameters $\tau_{1}$ and $\tau_{2}$ represent the timings of the step changes. Note that the initial stage with $u=0$ is the settling phase where the thermal profile is allowed to equilibrate.

The velocity ramp input is a piecewise linear input function and is characterised as:

$$
u(t)=\left\{\begin{array}{cc}
0 & 0 \leq t<\tau_{3} \\
\frac{\left(t-\tau_{3}\right)}{\left(\tau_{4}-\tau_{3}\right)} u_{3} & \tau_{3} \leq t<\tau_{4} . \\
u_{3} & t \geq \tau_{4}
\end{array}\right.
$$

The time parameter $\tau_{3}$ is the end of the settling phase and the beginning of the ramping phase. The parameter $\tau_{4}$ is the end time for the ramping phase where the pulling speed has reached the final value of $u_{3}$.

Table 2 gives the characteristic data for the pulling speed profiles for the step input and ramp input regimes.

Table 2: Pulling profile input data (corresponding to simulation results and discussions in Sections 4.2, 4.3, 5.2, and 5.3).

\begin{tabular}{llc}
\hline Regime & Pulling Parameter / (units) & Value \\
\hline Step Input & $u_{1} /(\mathrm{mm} / \mathrm{s})$ & 0.5 \\
& $\tau_{1} /(\mathrm{s})$ & 500 \\
& $u_{2} /(\mathrm{mm} / \mathrm{s})$ & 1.0 \\
& $\tau_{2} /(\mathrm{s})$ & 800 \\
Ramp Input & $u_{3} /(\mathrm{mm} / \mathrm{s})$ & 1.0 \\
& $\tau_{3} /(\mathrm{s})$ & 500 \\
& $\tau_{4} /(\mathrm{s})$ & 550 \\
\hline
\end{tabular}

Numerical data inputs to the model refer to the discretisation parameters. The temporal discretisation parameter is given as $\Delta t$. In the commercial model (as detailed in Section 2.3) the time step was set to $\Delta t=0.01 \mathrm{~s}$. For the non-commercial model (as detailed in Section 2.2) the time step was set to $\Delta t=0.001 \mathrm{~s}$. (For reasons of stability the non-commercial code required a lower time step.)

There are two spatial discretisation parameters, $\Delta x$ and $\Delta r$, which define the grid spacing in the axial and radial directions, respectively. The values for the discretisation parameters were 
selected following a grid sensitivity analysis. The analysis is given in the results section that follows.

\section{Results}

This section begins with a summary of the simulation results from the convergence exercise. This is followed by a comparison of the simulation results for each model. The demonstration of convergence in both models is a prerequisite to code-to-code verification. A successful convergence exercise demonstrates that the results are grid independent; comparison of the modelling results becomes meaningful only after convergence has been established. In this paper, the results from the commercial code form the benchmark data set that the noncommercial model is compared with. Comparisons between the commercial and noncommercial model results are done using the transient temperature data along the axis of the sample (i.e., at $r=0 \mathrm{~mm}$ ). Finally, the efficacy of the non-commercial model is demonstrated by showing results for the temperature distributions across the entire numerical domain at subsequent times in the simulation.

\subsection{Convergence results}

A grid sensitivity analysis was performed. Convergence test were carried out for both alloy compositions (Al-7wt.\%Si and Al-11wt.\%Si) using a double velocity step simulation case. Three control volume mesh densities were considered, equal spacing in the axial and radial directions were selected as $1 \mathrm{~mm}, 0.5 \mathrm{~mm}$, and $0.25 \mathrm{~mm}$. Discretization in time was set equal to the nominal settings given in Section 3.3. However, the non-commercial numerical codein which a fully explicit integration scheme is utilized-required shorter time discretization steps to fulfil the stability condition. Hence, for the case involving the densest grid the time step was reduced to $\Delta t=0.00025 \mathrm{~s}$. Divergence in results occurred using longer time integration steps, for example, with $\Delta t=0.001$ or $\Delta t=0.0005 \mathrm{~s}$.

Figure 3(a) and figure 3(b) show the comparison of results obtained for various mesh densities using the non-commercial model and the commercial model, respectively, in the case of the Al-7wt.\%Si alloy. For each results set, an initial time period (up to time $t=500 \mathrm{~s}$ ) is imposed where the alloy is stationary $(u=0 \mathrm{~mm} / \mathrm{s})$ and the temperatures are allowed to reach a steady-state equilibrium distribution. After this time, the pulling speed is abruptly increased to $0.5 \mathrm{~mm} / \mathrm{s}$ (at a time of $t=700 \mathrm{~s}$ ). At the end of this second stage, the pulling speed 
is increased to $1 \mathrm{~mm} / \mathrm{s}$ (until $t=900 \mathrm{~s}$ ). The results show good conformity. In figure 3(a) some low level of oscillations were observed, but only for the coarsest grid resolution.
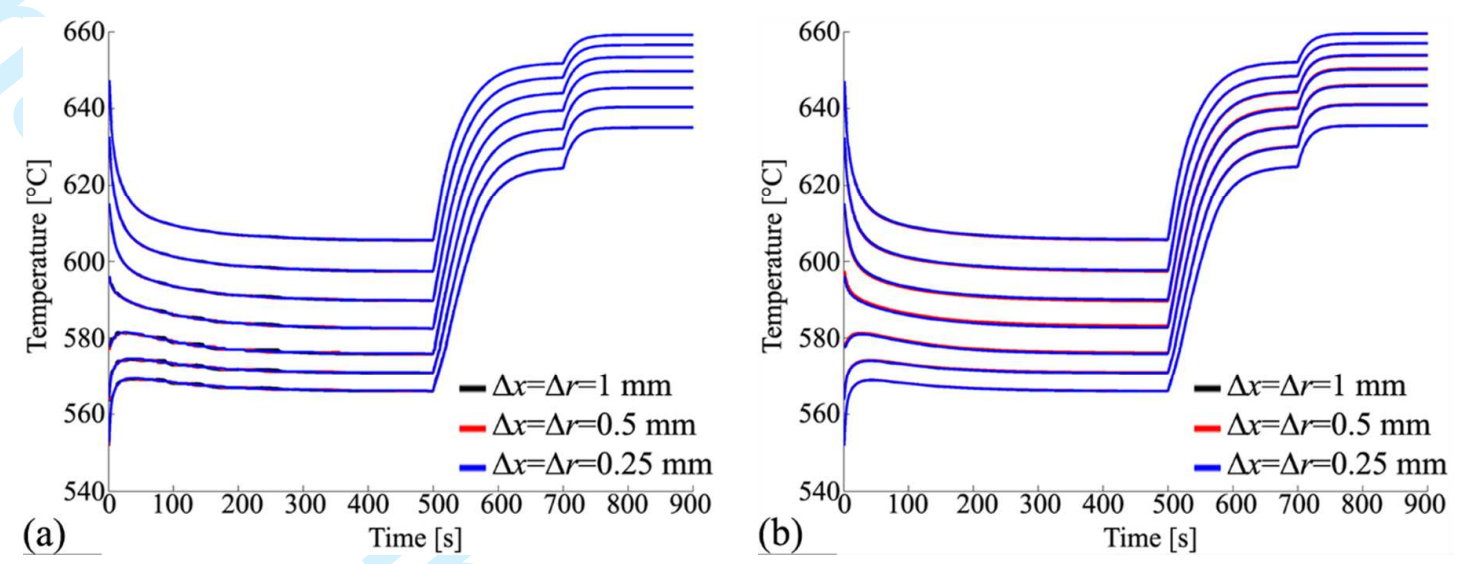

Figure 3: Temperature histories at several axial locations for the $\mathrm{Al}-7 \mathrm{wt} . \% \mathrm{Si}$ alloy with different grid spacing $(\Delta x=\Delta r)$ applied; (a) shows convergence results of the non-commercial model; (b) shows convergence results for the commercial model. Axial locations shown are: $x=63 \mathrm{~mm}$ (top), $67 \mathrm{~mm}, 71 \mathrm{~mm}$, $75 \mathrm{~mm}, 79 \mathrm{~mm}, 83 \mathrm{~mm}$, and $87 \mathrm{~mm}$ (bottom).

A similar convergence exercise was performed for the Al-11wt.\%Si alloy. Results are presented in a figure 4(a) for the non-commercial model and figure 4(b) for the commercial model.

(a)
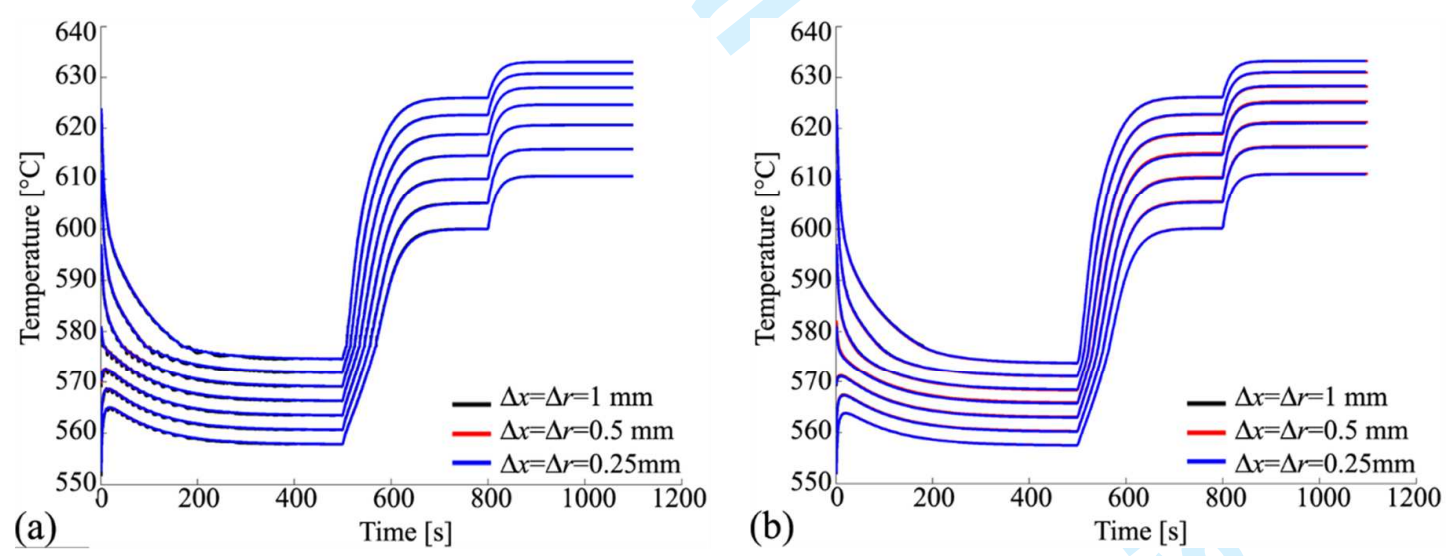

Figure 4: Temperature histories at several axial locations for the $\mathrm{Al}-11 \mathrm{wt} . \% \mathrm{Si}$ alloy with different grid spacing ( $\Delta x=\Delta r$ ) applied; (a) shows convergence results the non-commercial model; (b) shows convergence results for the commercial model. Axial locations shown are: $x=63 \mathrm{~mm}$ (top), $67 \mathrm{~mm}, 71 \mathrm{~mm}, 75 \mathrm{~mm}, 79$ $\mathrm{mm}, 83 \mathrm{~mm}$, and $87 \mathrm{~mm}$ (bottom).

\subsection{Comparison of numerical modelling results}

The foregoing simulation results show that the commercial and non-commercial models are grid convergent at or below the spatial grid resolutions specified. It is appropriate then to make comparisons between the two numerical models using like-for-like simulation scenarios. Simulation results are presented and compared (for both alloy compositions) next 
using each model. The results for the velocity step regime are considered first; this is followed by results for the velocity ramp regime.

\subsubsection{Comparison for velocity step regime}

Figure 5 shows the simulated thermal response histories for the two alloys at several positions along the axis of the sample. The results shown are in response to a double velocity step regime as specified in Table 2. Figure 5(a) shows the response for the sample with Al11wt.\%Si. Figure 5(b) shows the responses for the sample with Al-7wt\% Si.

(a)

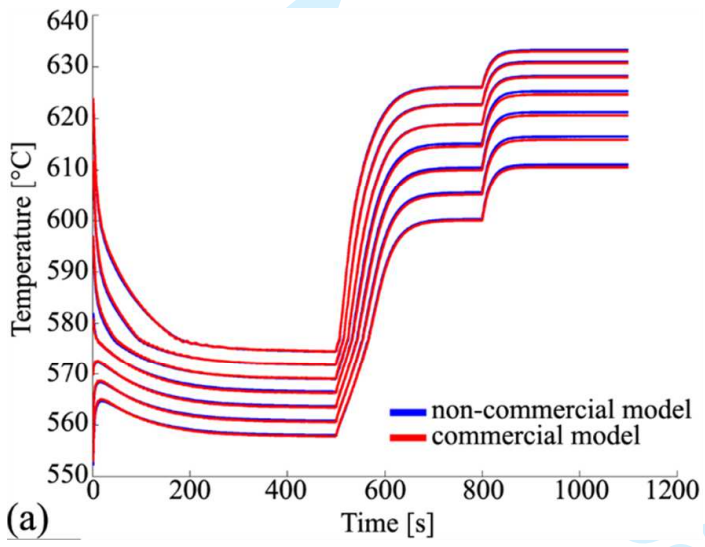

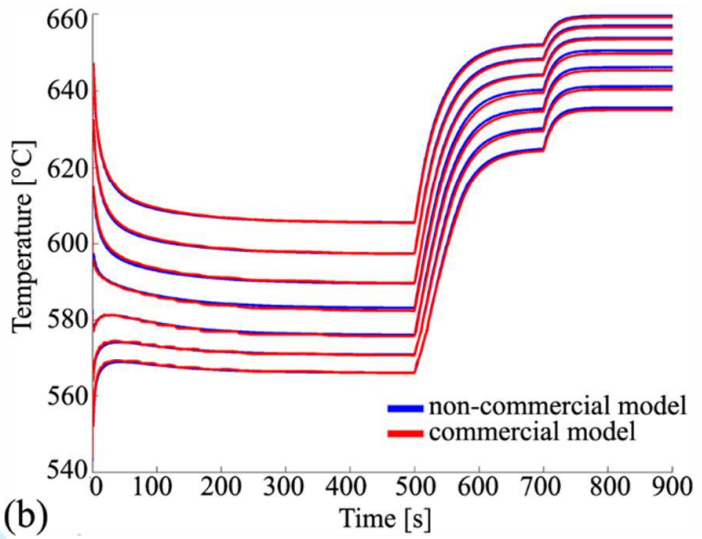

Figure 5: Temperature histories at several axial locations for (a) the Al-11wt.\%Si alloy and (b) the Al$7 \mathrm{wt} . \% \mathrm{Si}$ alloy in response to the velocity step regime specified in Table 2. Axial locations shown are: $x=63$ $\mathrm{mm}$ (top), $67 \mathrm{~mm}, 71 \mathrm{~mm}, 75 \mathrm{~mm}, 79 \mathrm{~mm}, 83 \mathrm{~mm}$, and $87 \mathrm{~mm}$ (bottom).

\subsubsection{Comparison for velocity ramp regime}

Figure 6 shows the simulated thermal response histories for the two alloys at several positions along the axis of the sample. The results shown are in response to the ramp input regime as specified in Table 2. Figure 6(a) shows the response for the sample with Al-11wt.\%Si. Figure 6(b) shows the responses for the sample with Al-7wt\% Si.
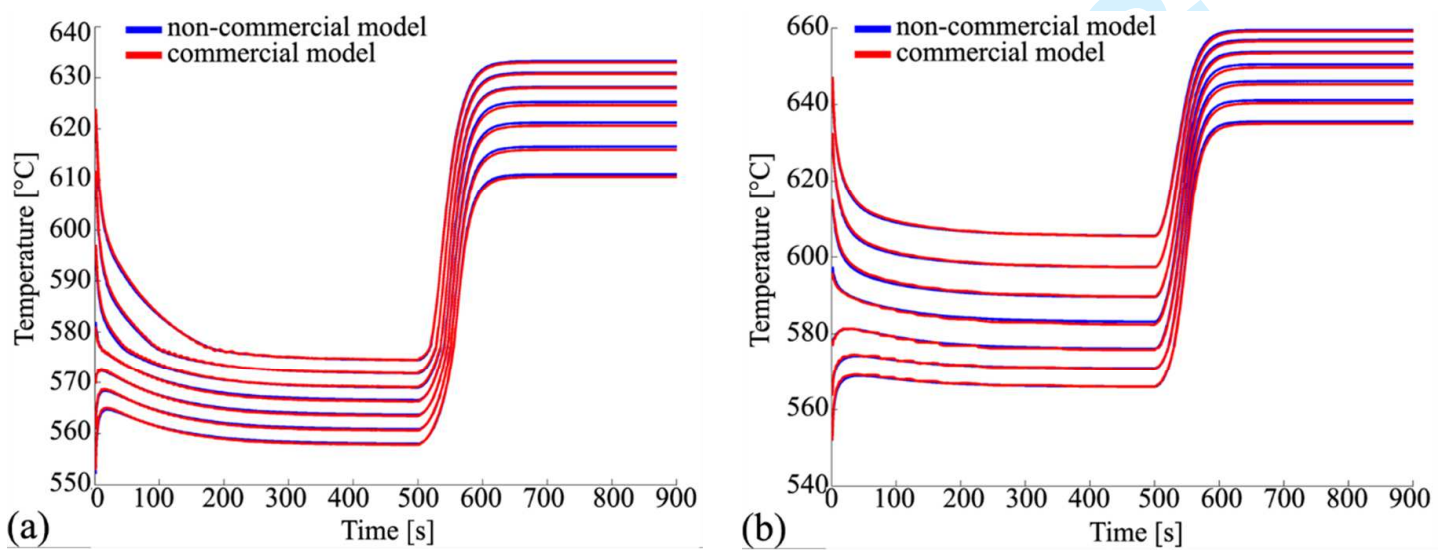

Figure 6: Temperature histories at several axial locations for (a) the Al-11wt.\%Si alloy and (b) the Al$7 \mathrm{wt} . \% \mathrm{Si}$ alloy in response to the velocity ramp regime specified in Table 2 . The red lines are the result for the non-commercial model; the blue lines are for the commercial model. Axial locations shown are: $x=63 \mathrm{~mm}$ 
(top), $67 \mathrm{~mm}, 71 \mathrm{~mm}, 75 \mathrm{~mm}, 79 \mathrm{~mm}, 83 \mathrm{~mm}$, and $87 \mathrm{~mm}$ (bottom).

The sample was motionless for the initial $500 \mathrm{~s}$. Then, over a $50 \mathrm{~s}$ period, the velocity was linearly increased to $1 \mathrm{~mm} / \mathrm{s}$. The system was then allowed stabilise by keeping the pulling velocity constant.

\subsection{Temperature distributions}

The predicted temperature distribution in the sample for the velocity step input regime, and considering the Al-11wt.\%Si alloy, is presented in figure 7. Figure 7(a) shows the temperature distribution at the end of the initial settling phase, at $t=500 \mathrm{~s}$, where the pulling velocity is zero. Figure 7(b) shows the temperatures at time, $t=800 \mathrm{~s}$, and figure 7 (c) shows the temperatures at time, $t=1100 \mathrm{~s}$. Considering the transient data presented earlier (in figure 5) it is assumed that the temperature distribution shown in figure 7 is steady. However, the pulling speeds were increased consecutively as per the data in Table 2. The equilibrium liquidus and eutectic isotherms lines are overlaid within this figure in heavy red and blue lines, respectively. The baffle zone is marked with vertical green lines (at axial positions of $0.06 \mathrm{~m}$ and $0.09 \mathrm{~m})$.

(a)

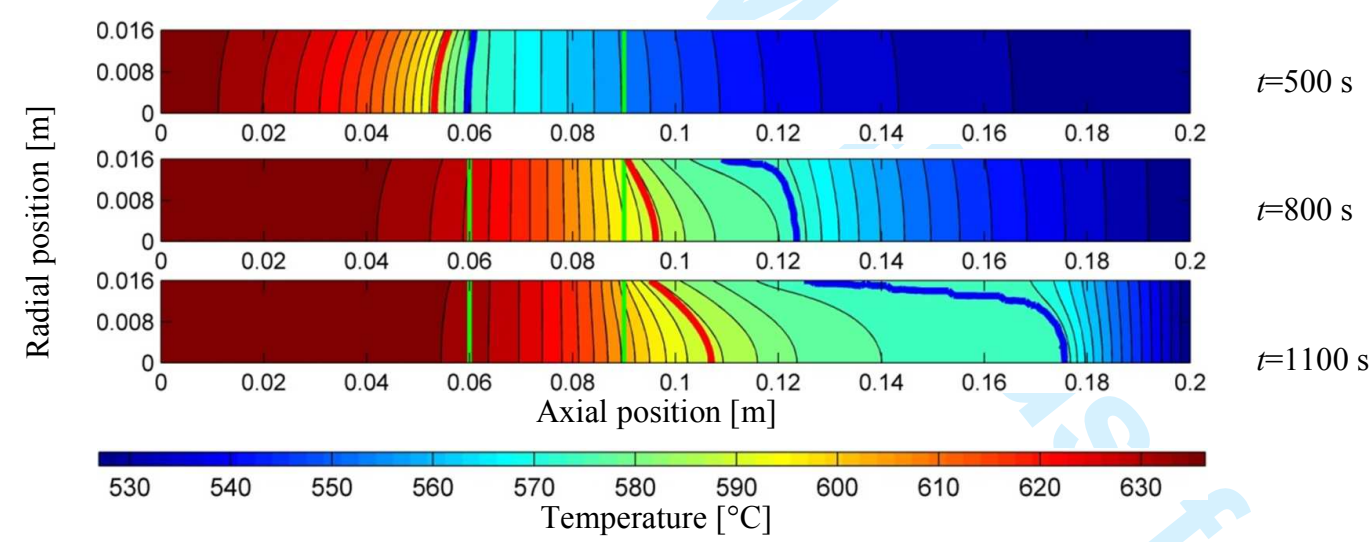

Figure 7: Simulated temperature distributions predicted using the non-commercial model for the $\mathrm{Al}-11 \mathrm{wt} . \% \mathrm{Si}$ alloy after: (a) $500 \mathrm{~s}$, (b) $800 \mathrm{~s}$ and (b) $1100 \mathrm{~s}$. The heavy red line gives the alloy equilibrium liquidus isotherms; the heavy blue line, the alloy eutectic isotherm. The vertical green lines are limits of the adiabatic baffle zone.

Figure 8 shows the corresponding temperature distributions for the Al-7wt.\%Si alloy under the same process conditions as that shown in figure 7. Mesh densities used in the results 
presented in figures 7 and 8 are equal to $0.5 \mathrm{~mm}$ in axial and radial directions. Both sets of results shown in these figures are produced using the non-commercial model.

(a)

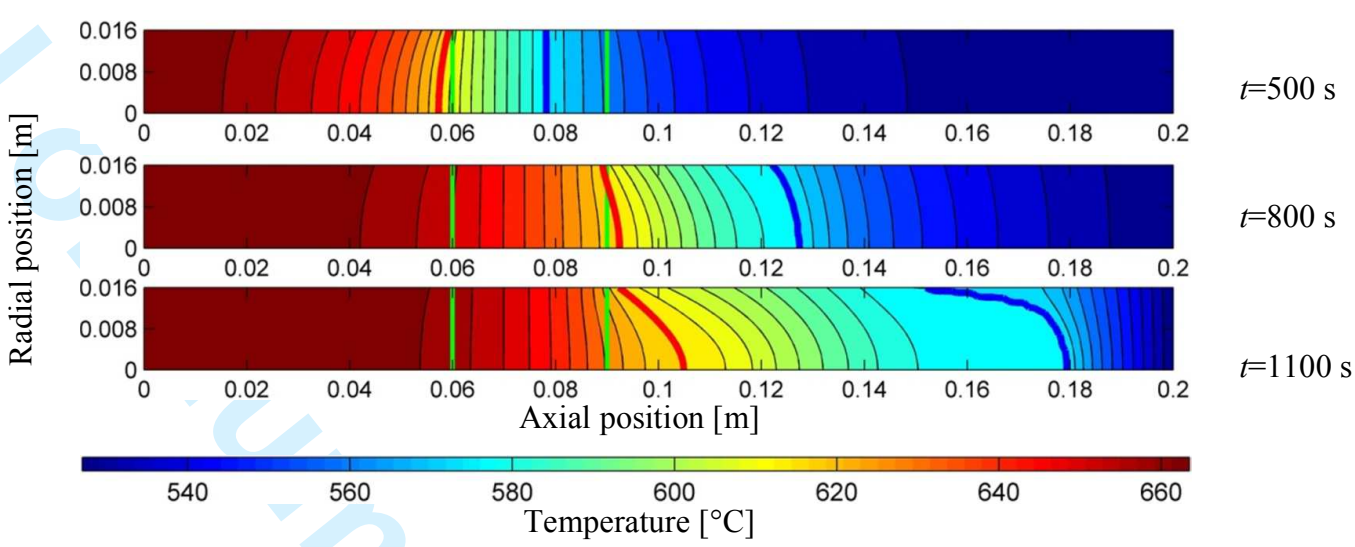

Figure 8: Simulated temperature distributions predicted using the non-commercial model for the $\underline{\mathrm{Al}-7 \mathrm{wt} . \% \mathrm{Si}}$ alloy after: (a) $500 \mathrm{~s}$, (b) $800 \mathrm{~s}$ and (b) $1100 \mathrm{~s}$. The heavy red line gives the alloy equilibrium liquidus isotherms; the heavy blue line, the alloy eutectic isotherm. The vertical green lines are limits of the adiabatic baffle zone.

\section{Discussion}

\subsection{Convergence results}

The results in Section 4.1 show that adequate convergence of results was achieved and grid independence was demonstrated. This is an important step in the presented analysis, since code-to-code verification involves comparison between two independent numerical models (both of which can suffer from numerical discretisation effects). It is noted that while the similarity achieved between the numerical results during grid doubling (in each of the respective models) is deemed to be acceptable, the results achieved for the Al-7wt.\%Si alloy are slightly better than that achieved in the Al-11wt.\%Si alloy.

The non-commercial model suffered from some numerical oscillations at the coarsest grid size tested. The time step required for the finest grid resolution had to be reduced to $0.000125 \mathrm{~s}$ for reasons of stability. Oscillations in temperature, shown in figure 4(a), observed in the initial settling period $(t<200 \mathrm{~s})$ for this mesh size disappeared with time.

\subsection{Comparison between models}

The two models showed good mutual agreement in their transient thermal responses. For the two alloys tested, and under the simulations conditions reported here, it is deemed that this 
level of agreement is acceptable for future work and further development of the noncommercial code.

\subsection{Temperature distributions (using the non-commercial model)}

The width of the mushy region is given by the distance between the blue and red isotherm lines in figure 7 and figure 8. It is expected that the alloy with the lower silicon composition will have a larger solidification interval (i.e., the temperature range over which solidification occurs, $T_{l}-T_{\text {eutectic }}$ ); the larger this interval, the wider the expected mush width. Clearly this is the case when comparing figure 7 , which shows a smaller mush width for the alloy with the lower solidification interval $\left(13{ }^{\circ} \mathrm{C}\right)$, when compared to figure 8 , which shows a larger mush width for the alloy with the higher solidification interval $\left(41^{\circ} \mathrm{C}\right)$. This is true for all cases (a), (b) and (c).

The isotherm lines in the adiabatic region are mostly vertical (as would be expected in an adiabatic region). Some radial heat flow is clearly occurring in the hot and cold regions of the domain since the isotherms there are slightly curved in the early part of the simulations, see part (a) of figure 7 and figure 8. As the pulling velocity is introduced, part (b) of figure 7 and figure 8 , the curvature of the isotherms is increased; this means that the radial heat flow contribution is increased in relation to axial heat flow contribution within the modelled domain. In the last stage of the simulation at maximum pulling velocity, part (c) of figure 7 and figure 8 , the curvature is further increased to the point where axial heat flow may be dominant over axial heat flow. Bridgman experiments (and in industry applications) are normally carried out so that solidification occurs within or near the adiabatic zone, thereby ensuring a near constant temperature gradient in the mushy region of the sample. Hypothetically, the results presented here would indicate to experiment designers that the mushy region is predicted outside the adiabatic zone, therefore a furnace (or furnace settings) redesign would be required.

The prediction of radial heat flow in Bridgman solidification is important since most solidification experiments, for example, studies concerned with columnar to equiaxed transition (Mooney, Hecht, et al., 2015), are designed such that directional solidification in the axial direction is desirable. This result also highlights the main benefit of the 2D Bridgman furnace model over its 1D predecessor (the BFFTM), since it can more realistically deal with radial heat flow in problems where the Biot number is greater than 0.1. 


\section{Conclusion}

The overall aims and objectives of this manuscript, given in Section 1.1, were achieved as follows:

1. An overview of the construction of a new (non-commercial) model for Bridgman furnace solidification was given.

2. An alternative model of the Bridgman process was developed to provide benchmark simulations for the purposes of a code-to-code verification exercise using the commercial software: ANSYS Fluent (commercial model).

3. A grid sensitivity analysis was performed for both the commercial and non-commercial models of solidification; mesh independence was demonstrated in each model.

4. The results from each model were then compared for velocity step input and velocity ramp input regimes (both of which deal with transient solidification), and using two different hypoeutectic compositions of Al-Si. Good conformity of results between the commercial and non-commercial models was achieved. The temperature histories predicted by simulation were coincident, or nearly coincident, in all cases.

5. The ability of the non-commercial model to deal with cases where significant radial heat transfer occurs in the sample was demonstrated. A large isotherm curvature was observed in the off-adiabatic region at high pulling speeds. Using the non-commercial model only, differences between simulations where the alloy composition changed were explained by the variance in the ratio of thermal conductivities of the liquid and solid phases, and by the difference in equilibrium liquidus temperature, for each alloy. The result demonstrates the usefulness of the new model to aid furnace and experiment design, particularly in applications where directional solidification in the axial direction is desired.

The work presented here is considered a confidence-building step in the development of the current model. Regarding future work, this model of Bridgman solidification does not consider the undercooled region ahead of the growing dendritic mushy region. The next step in the model development would be to implement a 'front tracking' approach ([Browne, D.J., Hunt, J.D., 2004, Banaszek, J., McFadden, S., Browne, D.J., Sturz, L., Zimmermann, G., 2007, Seredynski, M., Banaszek, J., 2010]) where an extent (or an envelope) of the growing columnar dendritic mush is traced on a fixed control volume grid, and equiaxed mushy zone nucleation and growth can be included in the simulation model. 


\section{Acknowledgements}

The work of the first author has been supported by the European Union in the framework of European Social Fund through the "Didactic Development Program of the Faculty of Power and Aeronautical Engineering of the Warsaw University of Technology".

Authors Battaglioli, Mooney and McFadden would like to acknowledge the European Space Agency PRODEX programme for funding this research (agreement numbers: 4000110385 and 4000107132) under the management of the Irish Space Delegation at Enterprise Ireland.

\section{References}

Banaszek, J., McFadden, S., Browne, D.J., Sturz, L., Zimmermann, G., 2007. Natural convection and columnar-to-equiaxed transition prediction in a front-tracking model of alloy solidification. Metall. Mater. Trans. A 38, 1476-1484.

Banaszek, J., Seredynski, M., 2012. The accuracy of a solid packing fraction model in recognizing zones of different dendritic structures. Int. J. Heat Mass Transf. 55, 43344339.

Battaglioli, S., Mooney, R.P., Seredyński, M., Robinson, A., Mcfadden, S., 2015. Computer Modelling of Manufacturing Processes: The development of a 2D axisymmetric model of the Bridgman casting process. IMC32.

Browne, D.J., Hunt, J.D., 2004. A fixed grid front-tracking model of the growth of a columnar front and an equiaxed grain during solidification of an alloy. Numer. Heat Transf. Part B Fundam. 45, 395-419.

Jung, H., Mangelinck-Noël, N., Nguyen-Thi, H., Billia, B., Reinhart, G., Buffet, A., 2009. Directional solidification processing on CET in Al-based alloys. Met. Mater. Int. 15, 2126.

Kartavykh, A.V., Ginkin, V.P., Ganina, S.M., 2014. Numerical modeling of power-down directional solidification process of $\mathrm{Ti}-46 \mathrm{Al}-8 \mathrm{Nb}$ refractory alloy. J. Alloys Compd. 586, S267-S273.

Łapka, P., Furmański, P., 2012. Fixed Cartesian grid based numerical model for solidification process of semi-transparent materials I: Modelling and verification. Int. J. Heat Mass Transf. 55, 4941-4952.

Liu, D.R., Mangelinck-Noël, N., Gandin, C.-A., Zimmermann, G., Sturz, L., Nguyen Thi, H., 
Billia, B., 2014. Structures in directionally solidified Al-7wt.\% Si alloys: Benchmark experiments under microgravity. Acta Mater. 64, 253-265.

McFadden, S., Browne, D.J., 2009. A front-tracking model to predict solidification macrostructures and columnar to equiaxed transitions in alloy castings. Appl. Math. Model. 33, 1397-1416.

McFadden, S., Browne, D.J., Gandin, C.A., 2009. A comparison of columnar-to-equiaxed transition prediction methods using simulation of the growing columnar front. Metall. Mater. Trans. A 40, 662-672.

Mooney, R.P., Hecht, U., Gabalcová, Z., Lapin, J., McFadden, S., 2015. Directional solidification of a TiAl alloy by combined Bridgman and power-down technique. Kov. Mater. 53, 187-917.

Mooney, R.P., Lapin, J., Klimová, A., McFadden, S., 2015. Conditions for CET in a gamma TiAl alloy. IOP Conf. Ser. Mater. Sci. Eng. 84, 012088.

Mooney, R.P., McFadden, S., 2014. Order verification of a Bridgman furnace front tracking model in steady state. Simul. Model. Pract. Theory 48, 24-34.

Mooney, R.P., McFadden, S., Gabalcová, Z., Lapin, J., 2014. An experimental-numerical method for estimating heat transfer in a Bridgman furnace. Appl. Therm. Eng. 67, 6171.

Mooney, R.P., McFadden, S., Rebow, M., Browne, D.J., 2012. A front tracking model for transient solidification of $\mathrm{Al}-7 \mathrm{wt} \% \mathrm{Si}$ in a Bridgman furnace. Trans. Indian Inst. Met. $65,527-530$.

Pelletier, D., Roache, P.J., 2000. Verification and Validation of Computational Heat Transfer. In: Minkowycz, W.J., Sparrow, E.M., Murty, J.Y. (Eds.), Handbook of Numerical Heat Transfer. John Wiley \& Sons, Inc., pp. 417-442.

Reinhart, G., Mangelinck-Noël, N., Nguyen-Thi, H., Schenk, T., Gastaldi, J., Billia, B., Pino, P., Härtwig, J., Baruchel, J., 2005. Investigation of columnar-equiaxed transition and equiaxed growth of aluminium based alloys by X-ray radiography. Mater. Sci. Eng. A 413-414, 384-388.

Rosch, W., Jesser, W., Debnam, W., Fripp, A., Woodell, G., Pendergrass, T.K., 1993. A technique for measuring the heat transfer coefficient inside a Bridgman furnace. J. Cryst. 
Growth 128, 1187-1192.

Scheil, E., 1942. Bemerkungen zur schichtkristallbildungle. Zeitschrift Fur Met. 70-72.

Seredynski, M., Banaszek, J., 2010. Front tracking based macroscopic calculations of columnar and equiaxed solidification of a binary alloy. J. Heat Transfer 132, 102301.

Timchenko, V., Chen, P.Y.P., Leonardi, E., De Vahl Davis, G., Abbaschian, R., 2000. A computational study of transient plane front solidification of alloys in a Bridgman apparatus under microgravity conditions. Int. J. Heat Mass Transf. 43, 963-980.

Timchenko, V., Chen, P.Y.P., Leonardi, E., de Vahl Davis, G., Abbaschian, R., 2002. A computational study of binary alloy solidification in the MEPHISTO experiment. Int. J. Heat Fluid Flow 23, 258-268.

Zhong, H., Li, S., Kou, H., Li, J., 2015. The solidification path related columnar-to-equiaxed transition in Ti-Al alloys. Intermetallics 59, 81-86. 


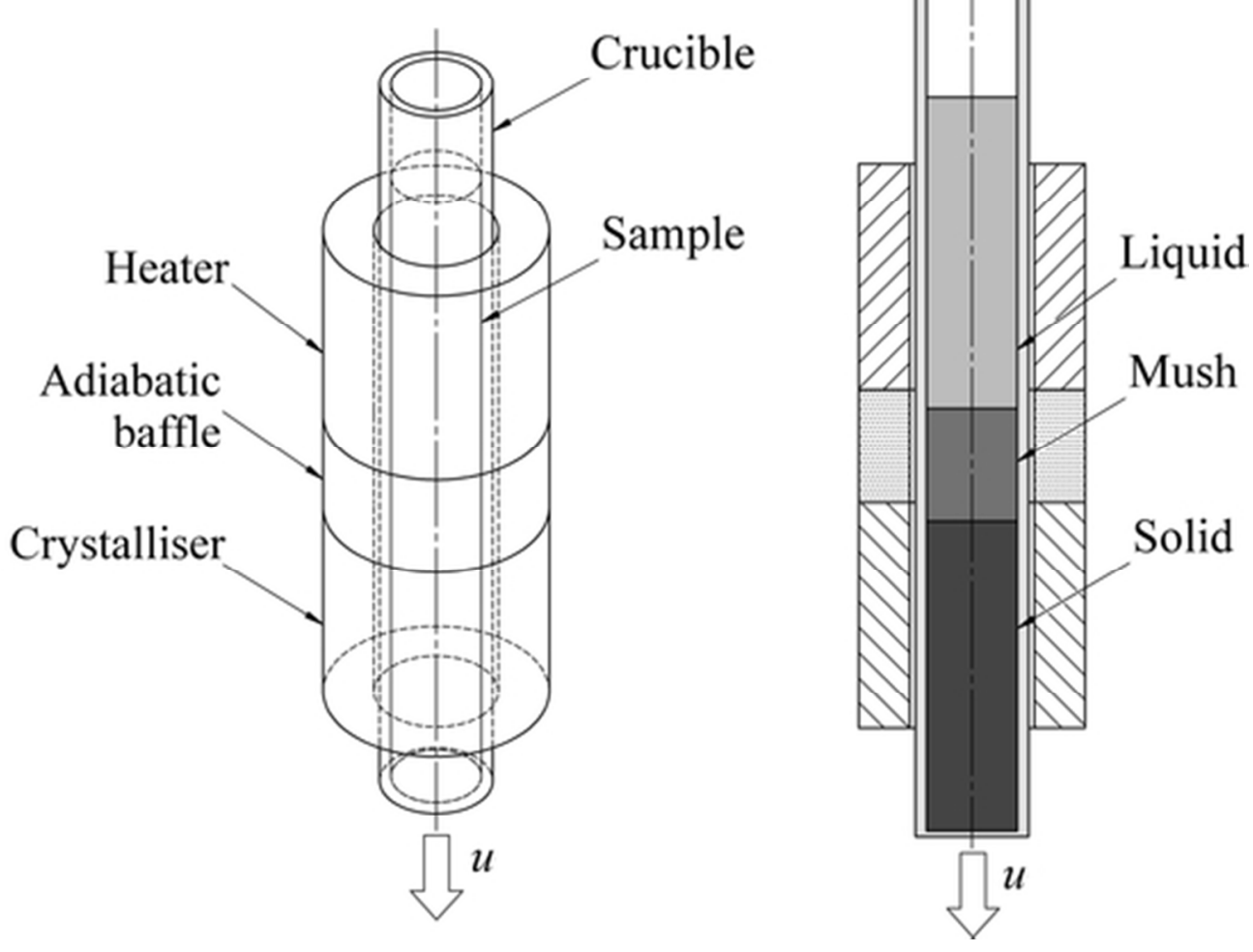

$93 \times 73 \mathrm{~mm}(150 \times 150 \mathrm{DPI})$ 


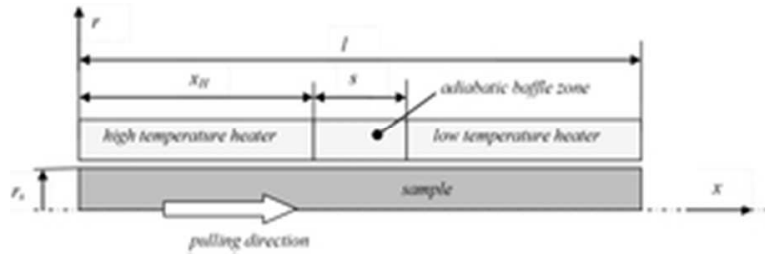

$49 \times 16 \mathrm{~mm}(150 \times 150 \mathrm{DPI})$ 

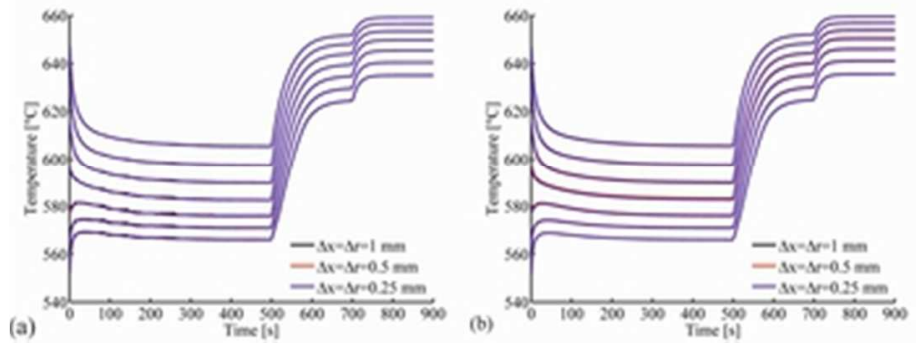

$60 \times 22 \mathrm{~mm}(150 \times 150 \mathrm{DPI})$ 

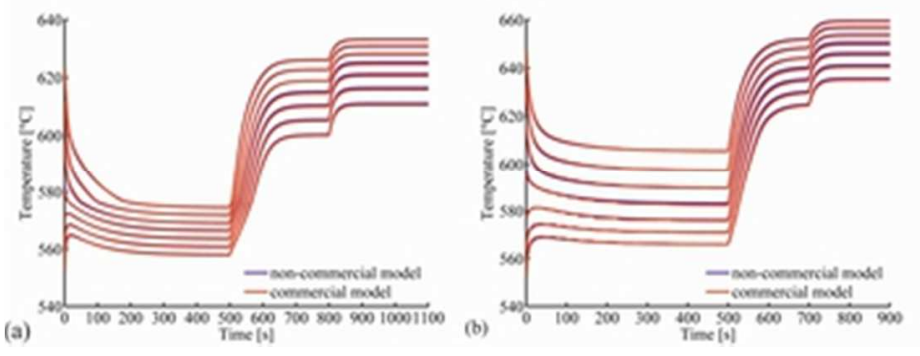

$60 \times 22 \mathrm{~mm}(150 \times 150 \mathrm{DPI})$ 


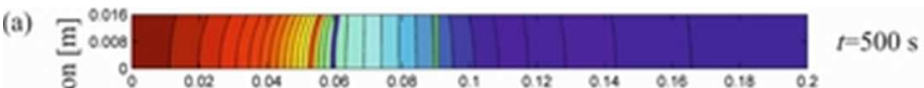

(b)

(c)

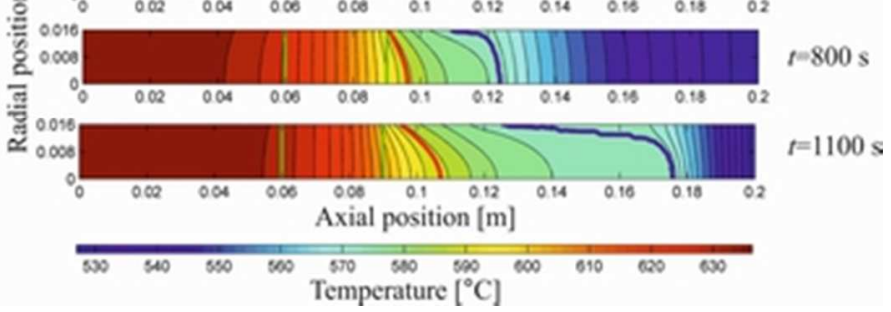

$60 \times 24 \mathrm{~mm}(150 \times 150 \mathrm{DPI})$ 
(a)

(b)

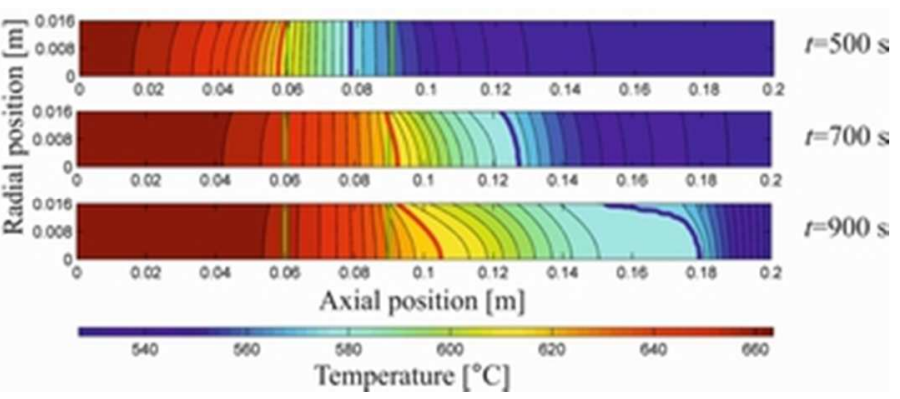

$61 \times 24 \mathrm{~mm}(150 \times 150 \mathrm{DPI})$ 